\title{
Discovering Serendipity
}

Ashna Nastar, University of Cambridge School of Clinical Medicine, Addenbrooke's Hospital, Cambridge, CB2 OSP, UK

Samira Afzal, University of Cambridge School of Clinical Medicine, Addenbrooke's Hospital, Cambridge, CB2 OSP, UK

\section{SRI LANKA- QUICK FACTS}

- Serendip - an ancient Arabic \& Persian name for Sri Lanka, the origin of 'serendipity' -the faculty of making fortunate discoveries by accident

- Population: 20.2 million

- Largest city: Colombo

- 11 185 rupees

- Language: Sinhala $74 \%$, Tamil $18 \%$, other $8 \%$. English is spoken by about $10 \%$ of the population.

- Life expectancy: 70 years (m), 78 years (f) (United Nations)

- Ayubowan means 'may you be blessed with long life'.

\section{KEY HEALTH ISSUE- The Dengue Epidemic}

Dengue is the most rapidly spreading mosquito-borne viral disease in the world, with an annual incidence of over 100 million infections (Malavige, 2006).

The disease is caused by four distinct, but closely related, virus strains all transmitted by the Aedes aegypti mosquito. Infection with one serotype provides life-long immunity to that virus but not to the others. Although primary prevention methods can significantly reduce the spread of disease, the incidence of dengue in Sri Lanka has unfortunately increased over the past ten years, with a total of 31046 cases and 227 deaths being reported between January and September 2010 alone (Sri Lankan Ministry Of Healthcare and Nutrition, 2010). The demographics of dengue fever have also evolved; a decade ago, children were predominantly affected, but recent years have seen a rise in the number of adult dengue patients, with significant morbidity and mortality. The reasons for this change are still unclear (Teixeira, 2008).

\section{Dengue Fever: Clinical Presentation}

Dengue is abrupt in onset, with a wide spectrum of clinical presentations often with unpredictable evolution and outcome. Usually, the patient experiences a high fever accompanied by severe headaches, incapacitating myalgias and arthralgias, periorbital pain, nausea and vomiting, and a typically maculopapular rash. The 
incubation period is 4-7 days. Symptoms usually last for 2-4 days, followed by a rapid drop in body temperature with profuse sweating. This precedes a period of normal temperature that lasts about a day. A second rapid rise in temperature follows. (Gibbons, 2002). Previous WHO classification grouped symptomatic dengue fever infections into three categories: undifferentiated fever, dengue fever (DF) and dengue haemorrhagic fever (DHF). Although this classification is still widely used, difficulties applying it in clinical situations have led to a revised criteria based on disease severity, which is hoped to improve decision-making concerning management strategies (WHO, 2009).

\section{Dengue Fever: Management}

Unfortunately, there is no treatment or vaccination available. Management is supportive with rest, oral rehydration or IV fluids (to expand plasma volume) and antipyretics for high fever (paracetamol [acetaminophen] but not aspirin, so that platelet function will not be impaired). Monitoring of blood pressure, haematocrit, platelet count, haemorrhagic manifestations, urinary output, and level of consciousness is important.

Dengue fever is an incapacitating disease, but prognosis is often favourable in previously healthy adults, as it is usually self-limiting with less than $1 \%$ case fatality (Alejandria, 2009). However, complications can occur.

\section{Dengue Fever: Complications}

At the extreme end of dengue's symptom spectrum are Dengue Haemorrhagic Fever (DHF) and Dengue Shock Syndrome, these typically occur in children under the age of 15 years. DHF and dengue shock usually develop around the third to seventh day of illness (WHO, 1997). Important risk factors for these complications include the virus strain and serotype, immune status of the host, age, and genetic predisposition (Alejandria, 2009). It is not certain what precipitates progression, although it has been suggested that previous infections with a different serotype of the virus predisposes to DHF when a person becomes re-infected(Halstead, 1981).

Classically, DHF has four clinical manifestations: high fever, haemorrhagic tendencies, hepatomegaly and circulatory disturbances. Moderate thrombocytopenia with concurrent haemoconcentration are distinctive clinical laboratory findings (WHO, 1997). The major pathophysiological hallmarks of DHF are plasma leakage (following increased vascular permeability) and abnormal haemostasis. The most common haemorrhagic feature is a positive tourniquet test which occurs in over $50 \%$ of patients ( A critical plasma volume loss leads to hypovolaemic shock, which creates the setting for the major causes of death in DHF. 
The common causes of death include disseminated intravascular coagulation (DIC), multi organ failure, massive bleeding, fluid overload (leading to cardiac failure or pulmonary oedema), and acute liver failure with encephalopathy.

Dengue Shock Syndrome is defined as DHF with signs of circulatory failure, including narrow pulse pressure $(\leq 20 \mathrm{~mm} \mathrm{Hg})$, hypotension, or frank shock.

Prognosis in dengue haemorrhagic fever and dengue shock syndrome depends on prevention, or early recognition and treatment of shock. Case fatality ranges from $2.5-5.0 \%$. Once shock sets in, fatality may be as high as $12-44 \%$ (Rigau-Perez, 1999).

At present, the mortality rate of dengue fever in Sri Lanka is 1\%, ten-fold higher than Thailand where it is only $0.1 \%$ (Ministry of Public Health, Thailand). This sizeable gap can be attributed to two main factors. Firstly, a significant proportion of Sri Lankan patients present late, often already displaying signs of shock. Secondly, poor fluid management within the hospital itself often leads to fluid overload and subsequent death. The optimal fluid regimen remains the subject of debate. One of the management difficulties is to correct hypovolaemia rapidly without precipitating fluid overload.

\section{Dengue Fever: The Future}

Public education is the key to better survival (WHO, 2000). This should emphasize effective elimination of mosquito breeding grounds and earlier recognition of dengue symptoms. However, as the epidemic rages, the need for a dengue vaccine has never been greater. Progress with the development of dengue vaccines has accelerated but, alongside this, the scale of the problem has rapidly grown and a safe effective vaccine remains an urgent worldwide priority. Several candidate vaccines have been developed that have shown promise in phase 2 clinical studies. Comparisons need to be made of different vaccination strategies and vector control programmes as well as the estimated cost-effectiveness of dengue prevention compared with potential treatment with new antiviral drugs (Webster, 2009). Many questions remain unanswered, and a coordinated effort is needed to confront the issue.

\section{References:}

1. Alejandria M.M., 2009. Dengue haemorrhagic fever or dengue shock syndrome in children. Clinical Evidence, 12, pii: 0917.

2. Epidemiology Unit, The Center for Prevention and Control of Communicable Diseases, Sri Lankan Ministry of Healthcare and Nutrition (2010). Distribution of Notified (H399) DF/DHF Cases\&Deaths - upto 1-10-2010[online]. Available at: [Accessed on 2nd October, 2010]

3. Gibbons R.V., Vaughn D.W., 2002. Dengue: an escalating problem. British Medical Journal, 324(7353), pp. 1563-1566. 
4. Halstead SB, 1981. The Pathogenesis of Dengue: Molecular Epidemiology in Infectious Disease. American Journal of Epidemiology, 114, pp 632-48.

5. Malavige G.N. et al., 2006. Patterns of disease among adults hospitalized with dengue infections. QJM - Monthly Journal of the Association of Physicians, 99 (5), pp. 299-305.

6. Ministry of Public Health, Thailand. Pro-Med Mail for International Society of Infectious Diseases(ISID), promed-mdbs@promedmail.org. Dengue-Thailand (02):Pattani from Bureau of Epidemiology, Ministry of Public Health, Thailand (published in Thai).[email] Pro-Med mail post to List members of ISID. Sent 26th May 2010. Available at[Accessed on 2nd October 2010]

7. National Travel Health Network and Centre, 2008. Travel Health Information Sheets: Dengue Fever [online] (updated November 2009) Available at: [Accessed on 20th September, 2010]

8. Rigau-Perez JG, et al., 1998. Dengue and dengue hemorrhagic fever. Lancet, 352, pp. 971-977. http://dx.doi.org/10.1016/S0140-6736(97)12483-7

9. Teixeira, M.G., 2008. Recent shift in age pattern of dengue hemorrhagic fever, Brazil. Emerging Infectious Diseseas, 14, pp. 1663. http://dx.doi.org/10.3201/eid1410.071164

10. Webster D.P., Farrar J. and Rowland-Jones S., 2009. Progress towards a dengue vaccine, Lancet Infectious Diseases, 9, pp. 678-87. http://dx.doi.org/10.1016/S1473-3099(09)70254-3

11. World Health Organisation, 1997. Dengue haemorrhagic fever: diagnosis, treatment, prevention and control. 2nd ed. Geneva: WHO Press.

12. World Health Organisation, 2009. Dengue: Guidelines for Diagnosis, Treatment, Prevention and Control - New Edition. Geneva: WHO Press.

13. World Health Organization, 2000. Report of the Informal Consultation: Strengthening implementation of the global strategy for dengue fever/dengue haemorrhagic fever prevention and control. Geneva, Switzerland, 18-20 October 1999. Geneva:WHO Press. 